\title{
Myxoid chondrosarcoma of the mandible in a 22-year-old man: A case report
}

\author{
YING CHEN and YING ZHANG \\ Department of Oral Surgery, Ninth People's Hospital, \\ Shanghai Jiaotong University School of Medicine, Shanghai 200011, P.R. China
}

Received November 20, 2015; Accepted June 2, 2016

DOI: $10.3892 / \mathrm{mco} .2016 .939$

\begin{abstract}
This study describes a case of myxoid chondrosarcoma of the mandible in a 22-year-old male patient. A tumour in the buccal gingiva of the lower left premolar region had been identified 2 years earlier. Whole-jaw panoramic radiographs showed a hypodense shadow in the mesiodistal area near the roots of teeth 34 and 35. A maxillofacial computed tomography scan revealed a mass in the lower left premolar soft tissue, with a shadow indicating bone destruction, a clear boundary and uniform density. The preliminary diagnosis at the outpatient department was 34-35 epulis. The patient underwent surgery for 34-35 gingival tumour resection, 34 and 35 extraction, and 34 and 35 immediate implantation. The postoperative pathological examination revealed a cellular type extraskeletal myxoid chondrosarcoma of the lower left mandible. Under general anaesthesia, the patient underwent lower left mandibular block and segmental resection, submandibular triangle dissection and vessel disassociation, and musculocutaneous flap repair in the oral and maxillofacial defect area. After 9 months of follow-up, the patient had no complaints of discomfort, and tumour recurrence was not observed on imaging examinations.
\end{abstract}

\section{Introduction}

Extraskeletal myxoid chondrosarcoma, also referred to as chondroid sarcoma, is a rare malignant soft tissue sarcoma that accounts for $2.5 \%$ of all soft tissue sarcomas and exhibits a multinodular structure that is rich in mucus (1). This type of tumor was first reported by Enzinger et al (2) in 1972 as a unique clinical case. Extraskeletal myxoid chondrosarcoma has the potential for multiple differentiation and was tradition-

Correspondence to: Professor Ying Zhang, Department of Oral Surgery, Ninth People's Hospital, Shanghai Jiaotong University School of Medicine, 1355 Yingchun Road, Shanghai 200011, P.R. China

E-mail: shdrzhangying@outlook.com

Key words: extraskeletal myxoid chondrosarcoma, mandible, soft tissue ally classified as a low-grade malignancy. However, long-term follow-up revealed that extraskeletal myxoid chondrosarcoma was associated with a high rate of local recurrence, metastasis and mortality. In 2005, it was categorised as a soft tissue tumour with uncertain differentiation by the WHO Classification of Soft Tissue Tumours (3). We herein report a case of mandibular extraskeletal myxoid chondrosarcoma and investigate its clinical and pathological characteristics, differential diagnosis, treatment and prognosis via a literature review.

\section{Case report}

This study was conducted according to the principles of the Declaration of Helsinki and approved by the Ethics Committee of the Shanghai Ninth People's Hospital. The images included in this article were captured by a hospital-based photographer at Shanghai Ninth People's Hospital, Shanghai Jiao Tong University School of Medicine. Permission to use these images for this study has been obtained from the patient.

A male patient, aged 22 years, had identified a gingival tumour in the lower left premolar region 2 years earlier. The tumor was $3 \times 2 \mathrm{~cm}$ in size, with no history of growth, pain or symptoms of numbness in the teeth or gums, and occasional bleeding. A clinical examination revealed a mass in the buccal gingiva of teeth 34 and 35 , sized $3 \times 2 \mathrm{~cm}$, with a dark red colour, a clear boundary and firm texture. The mass was not mobile and was not tender or painful on palpation. Whole-jaw panoramic radiographs revealed a hypodense shadow in the mesiodistal area near the root of teeth 34 and 35, with a clear boundary. A maxillofacial computed tomography (CT) scan revealed a mass in the lower left premolar soft tissue, with a shadow indicating bone destruction, a clear boundary and uniform density. The patient sought treatment in the Oral Surgery outpatient department of our hospital on December 17, 2014, complaining of a 'tumour in the lower left posterior gingiva with loosening of the teeth for $>2$ years'. The tumor was preliminarily diagnosed as 34-35 epulis in the outpatient department. Following discussion, a surgical plan was developed. On December 24, 2014, the patient underwent surgery for 34-35 gingival tumour resection, 34 and 35 extraction, and 34 and 35 immediate implantation. Intraoperatively, a cystic tumour was identified, with an intact capsule, no adhesions to the mandible, and a grey jelly-like surface on cross-section. A consultation regarding the postoperative pathological results 

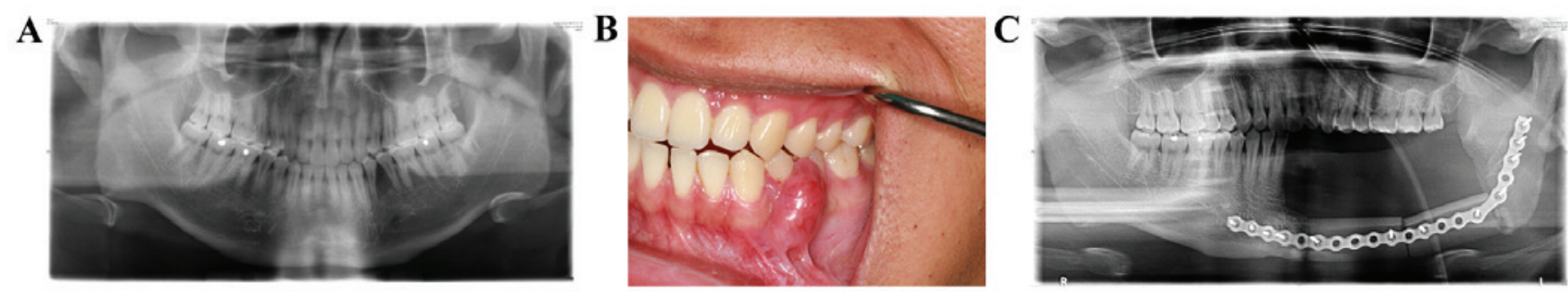

Figure 1. (A) Preoperative panoramic radiograph. A hypodense shadow is visible at the mesiodistal area near the root of tooth 34 and the mesiobuccal area of tooth 35. (B) Preoperative image of the patient's mouth. A buccal gingival tumour is visible (teeth 34 and 35 ), sized $3 \times 2 \mathrm{~cm}$, with a dark red colour. (C) Panoramic radiograph of the patient 1 week after surgery. Following lower left mandibular block and segmental resection, the positions of the bone graft and titanium were stable.
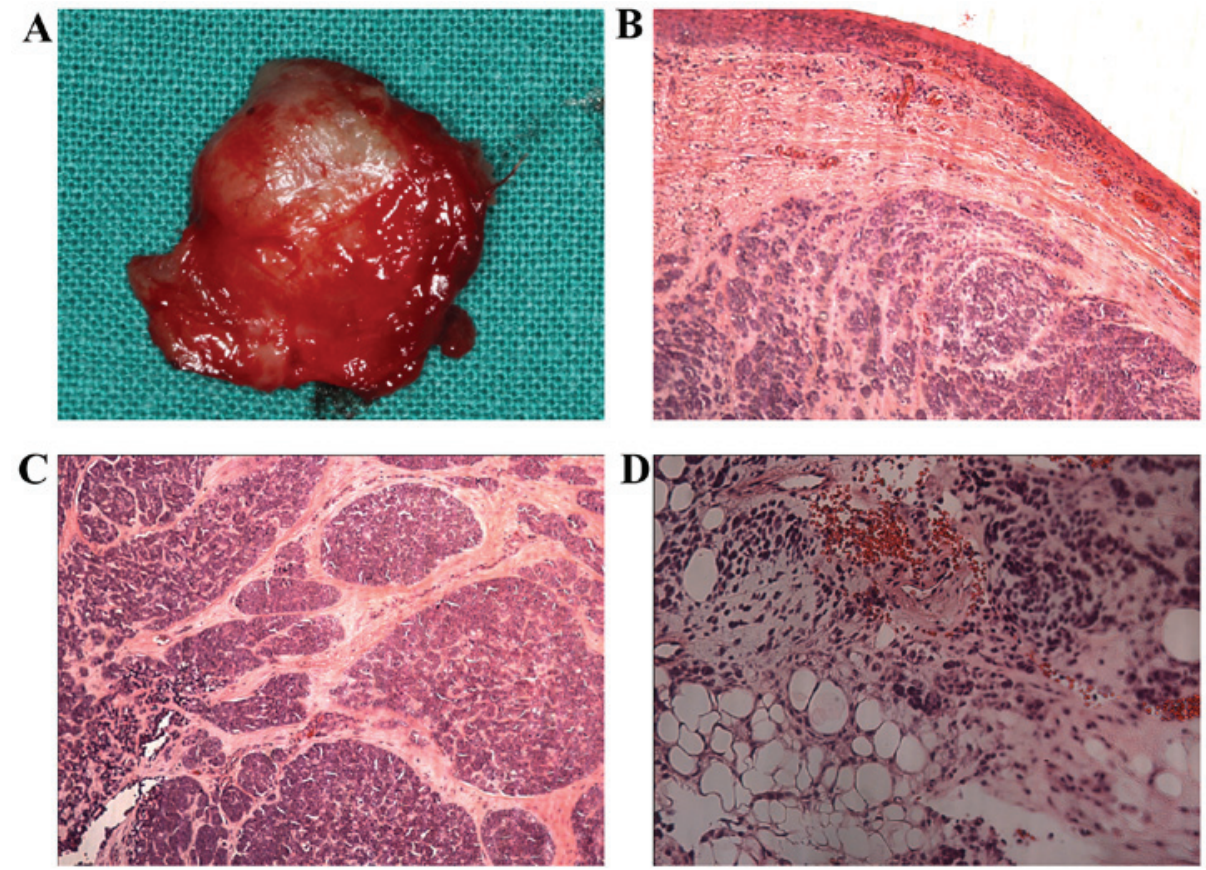

Figure 2. (A) Macroscopic image of the surgical specimen. The tumour was cystic, with a grey jelly-like surface. On microscopic examination under a light microscope (B) the tumour capsule was intact [hematoxylin and eosin (H\&E) staining; magnification, x100], (C) the tumour was divided into nodules by fibrous tissue bands (H\&E staining; magnification, x100) and (D) myxoid matrix was observed between cells (H\&E staining; magnification, x200).

was performed by experts in multiple hospitals, and the final diagnosis was cellular type extraskeletal myxoid chondrosarcoma of the lower left mandible. As extraskeletal myxoid chondrosarcoma is rare and exhibits uncertain differentiation, the patient was referred to the Department of Head and Neck Oncology of our hospital. On January 27, 2015, under general anaesthesia, the patient underwent lower left mandibular block and segmental resection, submandibular triangle dissection, vessel disassociation and musculocutaneous flap repair in the oral and maxillofacial defect area, titanium implantation in the mandibular defect, and fibular graft procurement. Symptomatic anti-inflammatory (Cephalosporins), anti-edematous (Prednisone) and hemostatic (ZhiKang; Chinese Traditional Medicine) treatment was provided postoperatively. The surgical specimen was fixed in $10 \%$ formalin, dehydrated, and embedded in paraffin to prepare conventional sections for haematoxylin and eosin staining and immunohistochemistry. The patient was followed up for 9 months, without complaints of discomfort. Tumour recurrence was not observed on whole-jaw panoramic radiographs and CT examination.
The pathological results revealed a mass sized $1.6 \times 1.5 \times 1.2 \mathrm{~cm}$ in the lower left mandible, with a pale colour and a cauliflower-like surface. The tumour cells exhibited nodular growth with a cartilage-like matrix. Some cells were heteromorphous, and mitotic activity was observed. The immunohistochemical markers showed positive staining for vimentin, CD99, S-100, protein gene product 9.5, glial fibrillary acidic protein and MYB and Ki67 ( 20\%), and showed negative staining for cytokeratin (CK)8, calponin, CK19, CAM5.2, CKp, P63, smooth muscle actin, CD34, epithelial membrane antigen (EMA), CD57 and chromogranin A (CgA). The pathological diagnosis was cellular type extraskeletal myxoid chondrosarcoma of the lower left mandible.

\section{Discussion}

Clinical manifestations. Extraskeletal myxoid chondrosarcoma often occurs in adults aged 50-60 years, with an average onset age of 52 years. Only few cases have been reported in children and adolescents (4). The male:female ratio is 2:1. 
Approximately $80 \%$ of the cases occur in the deep soft tissues of the proximal extremities and limb girdles and most commonly involve the thigh, which accounts for $\sim 69 \%$ of the cases. Approximately $20 \%$ of the cases are located in the trunk, mainly in skeletal muscles, tendons and the deep subcutis; a few cases involve the skin and bone tissue, whereas rare sites include the vulva and the breast (5). In recent years, individual cases occurring intracranially (hypothalamus) and in the buccal mucosa have been reported (6,7). According to a review of the literature, no case of extraskeletal myxoid chondrosarcoma occurring in the mandible has been reported to date. The main clinical manifestation is a slow-growing tumour; local pain and dysfunction may be present in some cases. Tumours located in joints may limit movement; tumours located in the brain may cause headaches or visual disorders, whereas tumours located more superficially may lead to an increase in skin tension, superficial varicose veins, or skin ulcers. Tumour sizes range from 1 to $25 \mathrm{~cm}$, with an average size of $7 \mathrm{~cm}$, and have a nodular appearance with a clear boundary.

Pathological characteristics. As discussed in Oliveira et al, the surface of extraskeletal myxoid chondrosarcomas is myxoid or jelly-like, with a localised myxoid-like transparent appearance; cysts, haemorrhage, or necrosis may be observed. Under a light microscope, extraskeletal myxoid chondrosarcomas usually exhibit a nodular structure with abundant myxoid stroma (8). The tumour cells are circular or polygonal and arranged in a strip-like, sieve-like and fibrous network structures, with abundant cytoplasm that is strongly eosinophilic or lightly stained. The nuclei are relatively uniform, with small and inconspicuous nucleoli, and the mitotic figures are 2-3/10 high-power fields (HPFs) (8). In the present case, immunohistochemical examination of the tumour revealed that vimentin was continuously expressed, with S-100 and EMA focally expressed in several lesions, whereas neurofilament protein, neuron-specific enolase and $\mathrm{CgA}$ are expressed in cases with neuroendocrine differentiation.

\section{Differential diagnosis}

Epulis. As discussed in Kfir et al, epulis is more common among young and middle-aged individuals and female patients. It often occurs in the buccal labial gingival papillae and most commonly in the premolar area (9). The tumour is relatively limited, varying in size, usually spherical or elliptical, and may sometimes be lobulated, polypoid-like, or have a broad sessile base. Vascular and granulomatous type tumours are soft and red; fibrous type tumours have a firm and hard texture with a pink colour. Generally, the tumour grows slowly and is usually pain-free, unless ulceration occurs on the tumour surface. The pathological characteristics of granulomatous epulis include newly generated capillaries and fibroblasts accompanied by inflammatory cell infiltration, mainly consisting of lymphocytes and plasma cells, and the gingival epithelium often shows false intraepithelial hyperplasia. Pathologically, fibrous type epulis exhibits fibrosis of the granulation tissue, with a reduction in the cellular and vascular components (9). In the present case, a small amount of chronic inflammatory cell infiltration was observed in the coarse collagen fibres, which may calcify or ossify within the fibre bundle, presenting as irregular trabecular bone without dental epithelial structures.
Haemangioma. As discussed in Wyke, haemangiomas that occur in the oral and maxillofacial area account for $60 \%$ of all haemangiomas in the body (10). They are mostly located on the facial skin, subcutaneous tissue and oral mucosa, such as the tissues of the tongue, lips and floor of the mouth, while a few cases have been reported in the jaw or deep tissues. Cavernous haemangioma is a slow-growing soft mass with no subjective symptoms. When the head is in a low position, the tumour expands due to hyperaemia, and if the head returns to the normal position, the shape of the tumour is restored. Superficial tumours produce a blue-purple colour on the skin surface or mucous membranes. The skin colour is normal when the tumour is located deeper. The tumour is soft on palpation, with no clear boundary and no tenderness. The tumour size is decreased by extrusion and returns to the original size after the pressure is released. The histopathological characteristics of the tumour include the presence of irregular cavities in the lower part of the dermis and the subcutaneous tissue, which contains red blood cells and fibrous tissue. The cavity wall is a monolayer of endothelial cells. Endothelial cell hyperplasia may be observed in the larger vascular compartment, resulting in a thickened wall (10).

Fibrosarcoma. As discussed in Prat and Scully (11), fibrosarcoma is common in young adults. Tumours located in the mouth and jaw are usually found in the gum and the surface of the jaw, have a spherical or lobulated shape and exhibit a purple-red colour, often with ulceration and haemorrhage. Invasion of the surrounding tissue may lead to bone destruction and loosening of the teeth or tooth loss. Peripheral type tumours may present as a local mass in the early stage, followed by pain. On histopathological examination, the tumour is composed of spindle fibroblasts and most skin fibrosarcomas are well-differentiated, with few mitoses, no obvious spiral structures and reduced fibre generation. When these phenomena are present, they indicate that the tumour is invasive and the prognosis is poor.

Treatment and prognosis. As the prognosis of extraskeletal myxoid chondrosarcoma is good, most scholars originally classified it as a low-grade malignancy. However, long-term follow-up revealed that, although the behaviour of this tumour is inert, it was associated with a high rate of local recurrence, metastasis and mortality. The local recurrence rate was $48 \%$, and in such cases, $58 \%$ of tumours exhibited multifocal recurrence. The metastasis rate was $46 \%$, and the most common metastatic site was the lung, accounting for $63 \%$ of the cases; however, extrapulmonary metastasis and diffuse metastasis may also occur (12). It has been reported that the 5-, 10- and 15 -year survival rates are 82, 65 and 58\%, respectively (13). The adverse factors for poor prognosis of extraskeletal myxoid chondrosarcoma include a tumour diameter $>10 \mathrm{~cm}$, abundant cells, tumour atypia, a mitotic figure count $>2 / 10$ HPFs, and tumour metastasis.

Extraskeletal myxoid chondrosarcoma is refractory to radiotherapy and chemotherapy $(14,15)$. Wide surgical resection is currently the preferred treatment, and incomplete tumour resection is considered a major cause of recurrence. Postoperative localised radiotherapy and systematic chemotherapy may be applied to reduce the rate of recurrence and 
metastasis. Currently, the optimal chemotherapy regimen for extraskeletal myxoid chondrosarcoma has not been determined and further investigation is required in this field. In recent years, with the development of gene therapy, researchers have found that regulating the specific proteins of extraskeletal myxoid chondrosarcoma may be a potential treatment, but this method remains in the clinical trial stage (16).

\section{References}

1. Meis-Kindblom JM, Bergh P, Gunterberg B and Kindblom LG: Extraskeletal myxoid chondrosarcoma: A reappraisal of its morphologic spectrum and prognostic factors based on 117 cases. Am J Surg Pathol 23: 636-650, 1999.

2. Enzinger FM and Shiraki M: Extraskeletal myxoid chondrosarcoma. An analysis of 34 cases. Hum Pathol 3: 421-435, 1972.

3. Fletcher CDM, Bridge JA, Hogendoorn P and Mertens F (eds): WHO classification of tumours of soft tissue and bone. In: WHO Classification of Tumours. Volume 5. 4th edition. IARC press, Lyon, pp223-224, 2013.

4. Yi JW, Park YK, Choi YM, Hong HP and Chang SG: Bulbous urethra involved in perineal extraskeletal myxoid chondrosarcoma in a child. Int J Urol 11: 436-439, 2004.

5. Fotiadis C, Charalambopoulos A, Chatzikoklis S, Zografos GC, Genetzakis $M$ and Tringidou R: Extraskeletal myxoid chondrosarcoma metastatic to the pancreas: A case report. World J Gastroenterol 11: 2203-2205, 2005.

6. Angiero F: Extraskeletal myxoid chondrosarcoma of the left buccal mucosa. Anticancer Res 32: 3345-3350, 2012.

7. Park JH, Kim MJ, Kim CJ and Kim JH: Intracranial extraskeletal myxoid chondrosarcoma: Case report and literature review. J Korean Neurosurg Soc 52: 246-249, 2012.
8. Oliveira AM, Sebo TJ, McGrory JE, Gaffey TA, Rock MG and Nascimento AG: Extraskeletal myxoid chondrosarcoma: a clinicopathologic, immunohistochemical, and ploidy analysis of 23 cases. Mod Pathol 13:900-909, 2000.

9. Kfir Y, Buchner A and Hansen LS: Reactive lesions of the gingiva. A clinicopathological study of 741 cases. J Periodontol 51: 655-661, 1980.

10. Wyke BD: Primary hemangioma of skull rare cranial tumor review of the literature and report of case with special reference to roentgenographic appearance. Am J Roentgenol Radium Ther 61: 302-316, 1949.

11. Prat $\mathbf{J}$ and Scully RE: Cellular fibromas and fibrosarcomas of the ovary: a comparative clinicopathologic analysis of seventeen cases. Cancer 47: 2663-2670, 1981

12. Gupta R, Sharma MC, Attri S and Guleria S: Testicular metastasis of extraskeletal myxoid chondrosarcoma: Report of first case. Urology 71: 984.e1-e4, 2008.

13. Drilon AD, Popat S, Bhuchar G, D'Adamo DR, Keohan ML, Fisher C, Antonescu CR, Singer S, Brennan MF, Judson I and Maki RG: Extraskeletal myxoid chondrosarcoma: A retrospective review from 2 referral centers emphasizing long-term outcomes with surgery and chemotherapy. Cancer 113: 3364-3371, 2008.

14. Stacchiotti S, Dagrada CP, Morosi C, Negri T, Romanini A, Pilotti S, Gronchi A and Casali PG: Extraskeletal myxoid chondrosarcoma: Tumor response to sunitinib. Clin Sarcoma Res 2: 22, 2012.

15. Han K, Sun YJ, Shan Z, Zhang JJ, Lin F, Zhao H, Meerani S and Yao Y: Extraskeletal myxoid chondrosarcoma: A case report of complete remission by chemotherapy and review of the literature. BMJ Case Rep 2010: bcr07.2009.2128, 2010.

16. Stacchiotti S, Dagrada GP, Morosi C, Negri T, Romanini A, Pilotti S, Gronchi A and Casali PG: Extraskeletal myxoid chondrosarcoma: Tumor response to sunitinib. Clin Sarcoma Res 2: 22-33, 2012. 